\title{
Outcome of Laser Urethrotomy in Comparison with Optical Internal Urethrotomy in Recurrent Stricture Urethra Following Perineal Anastomotic Urethroplasty for PUDD
}

\author{
Ripan Debnath ${ }^{1}$, Md. Nabid Alam ${ }^{2}$, Md. Towhid Belal ${ }^{3}$, Prodyut Kumar Saha ${ }^{4}$, Uttam Karmaker ${ }^{5}$, \\ Md. Shafiqul Alam Chowdhury ${ }^{6}$
}

Received: 19 - 07 - 2020 Accepted: 13 - 09 - 2020 Conflicts of interest: None

Keywords: Stricture urethra, PUDD, laser urethrotomy, optical internal urethrotmy (OIU), RGU\&MCU

\begin{abstract}
Objective: To compare the outcome of laser urethrotomy and optical internal urethrotomy(OIU) for the treatment of recurrent stricture urethra following perineal anastomotic urethroplasty for posterior urethral distraction defect.

Materials and methods: The study was conducted in Dhaka Medical College Hospital from January 2013 to December 2014.Male patients presented with obstructed voiding symptoms following perineal anastomotic urethroplasty were evaluated by their history, physical findings and investigations (urinalysis, uroflowmetry, retrograde urethrogram and micturiting cystourethrogram ) \& primarily 64 patients are selected by purposive sampling. Patients are divieded again by random allocation into group $A$ and group $B$ and underwent for OIU and laser urethrotomy respectively.
\end{abstract}

Results: Comparison was made to find out the better method between optical urethrotomy and laser urethrotomy. Overall per-operative complications (bleeding, extravasations of irrigating fluid, false passage and broken knife) in the former group were $31.3 \%$ compared to none in the latter group. Post-operative complications like bleeding, haematoma, penile oedema and erectile dysfunction were found only in Group-A ( $p=0.002)$. The mean duration of postoperative catheterization and average hospital stay were observed to be much higher in Group-A than that in Group-B ( $p=0.000008 ; p=0.0006)$. Comparison of final outcome (development of stricture) between groups at 1 year of evaluation in Group- $A$ and Group-B was not significant $(p=0.320)$.

Conclusion: Laser urethrotomy is better than optical urethrotmy in regards of peroperative and post-operative complications.

1. Assistant Professor, Department of Urology, Dhaka Medical College \& Hospital, Dhaka.

2. Medical Officer, Department of Urology, Banghabandhu Sheikh Mujib Medical University, Dhaka.

3. Assist. Professor, Department of Urology, Dhaka Medical College \& Hospital, Dhaka.

4. Associate Professor, Department of Urology, Dhaka Medical College \& Hospital, Dhaka.

5. Associate Professor, Department of Urology, Dhaka Medical College \& Hospital, Dhaka.

6. Professor of Urology \& Vice-Principal, Dhaka Medical College , Dhaka.

Correspondence: Dr. Ripan Debnath, Assistant Professor, Department of Urology, Dhaka Medical College \& Hospital, Dhaka-1000, Bangladesh. E-mail: ripandebnath1234@gmail.com 


\section{Introduction}

Of all injuries to the urinary tract the most debilitating is that which affects the posterior urethra. Posterior urethral distraction defect (PUDD) is a challenging urologic problem due to potentially extensive fibrotic response to urinary extravasation. If not managed properly it may lead to a lifelong disabling condition comprising not only inability to void but also inability to reproduce and recurrent stricture after anastomotic urethroplasty occurs in about $15 \%$ of cases ${ }^{1}$. Most of which need redo surgery which is also much more difficult.But many of these can be successfully corrected by endoscopic manuevre- either optical internal urethrotomy(OIU) or by laser core through . The results of these endoscopic treatment are durable in most cases and most authors accept these cases as successful urethroplasty. The data describing the outcome of visual internal urethrotomy(VIU) in the setting of recurrent strictures after anastomotic urethroplasty are scarce. Endoscopic management of recurrent anastomotic strictures become more successful where there is short stricture and a decrease in periurethral fibrosis after excision of the fibrotic segment during urethroplasty ${ }^{2}$. They offer definite advantages, such as easy to perform, short hospital stay, low morbidity and fewer complications and costeffective. Internal urethrotomy refers to any procedure that opens the stricture by incising or ablating it transurethrally. As a curative modality internal urethrotomy should be limited to mucosal fold i.e. stricture with an iris like configuration and with only superficial spongiofibrosis. Incision must be limited through the depth of spongiofibrosis disrupting the soft scar so that underlying soft elastic tissue can allow expansion of urethral lumen. Internal urethrotomy does not produce an epithelial approximation rather aims to separate the scarred epithelium, so healing occurs by secondary intention, wound contraction closes the wound defect and limits the size of area that requires epithelialization. If epithelialization progress completely before wound contraction, then internal urethrotomy will be successful. If contraction significantly narrows the lumen before epithelialization then stricture can recur.The most common complication of internal urethrotomy is recurrence of stricture; less commonly noted complications are bleeding and erectile dysfunction ${ }^{3}$. Although early results were encouraging but recent studies revealed less promising long-term results with a failure rate of up to $68 \%^{4}$. Their high recurrence rate has led to some urologist to develop new endoscopic techniques, which could achieve long term better results. One of these is laser technology; The advantage of the laser treatment is that scar tissue can be vaporized and removed after cutting. The reason of using various types of lasers is decreased formation of scar. Reportedly, the success rate of laser treatment ranges between $36 \%$ and $50 \%{ }^{5,6}$. The development of recurrent stenosis after laser incision is largely affected by the wavelength of the laser, namely, absorption of the energy by tissues and the thermal effect on surrounding tissues. The absorption by tissues is deepest ( 4 to $5 \mathrm{~mm}$ ) with Nd:YAG laser. The Ho:YAG laser displays shallowest absorption $(<0.5 \mathrm{~mm})$. Thus, this laser has the least effect on surrounding tissues and is presumed to induce less scar tissue formation. The Ho: YAG laser is the most recent type of laser added to the urologist's armamentarium. The present study is designed to observe the outcome of the cold knife optical urethrotomy and Ho:YAG laser urethrotomy for the treatment of recurrent stricture urethra following perineal anastomotic urethroplasty for posterior urethral distraction defect.

\section{Hypothesis}

Laser urethrotomy is better than optical urethrotomy for the treatment of recurrent stricture urethra following perineal anastomotic urethroplasty for posterior urethral distraction defect.

\section{Objectives}

To compare the outcome of laser urethrotomy and optical urethrotomy for the treatment of recurrent stricture urethra following perineal anastomotic urethroplasty for posterior urethral distraction defect

\section{Materials and methods}

This is an experimental study conducted in the Dept. Of Urology, Dhaka Medical College Hospital (DMCH) between January 2013 to December 2014.Study population included the. male patients presented with obstructed voiding symptoms following perineal anastomotic urethroplasty for PUDD attending at the Urology OPD of DMCH. Patients with recurrent stricture urethra following perineal anastomotic urethroplasty for PUDD are included in the study but Patients previously treated with dilatation or urerthrotomy, stricture segment $\tilde{A} 1 \mathrm{~cm}$, age more than 70 yrs were excluded. The sample size was determined 
using apropiate formula and finally 64 patients were recruited by purposive sampling. Male patients presented with obstructed voiding symptoms following perineal anastomotic urethroplasty attending at the Urology OPD of DMCH were evaluated by their history, physical findings and investigations (urinalysis, uroflowmetry, retrograde urethrogram and micturiting cystourethrogram (RGU $\& \mathrm{MCU})$.All the patients ranging from $15-70$ years were divided into two group by random allocation procedure and were numbered chronologically and odd number grouped as Group-A and even number as Group-B.Group-A included 32 cases and treated by optical internal urethrotomy. Group-B included 32 cases and treated by laser urethrotomy A structured questionnaire (research instrument) were completed which include patent's name, age, address, chief complains, duration of disease. Careful local, general and systemic examinations were carried out. All baseline investigations for anesthetic checkup were done. All operations were done under spinal anaesthesia in modified lithotomy position A broad spectrum antibiotic was given preoperatively prior to induction of anaesthesia in every case. Optical internal urerthrotomy were performed with Storz-Sachse cold knife optical urethrotome, using 0 degree telescope. The instrument was inserted into the urethra and the stricture was visualized. A guide wire was passed across the stricture in order to show the line of urethra beyond and to guide the urethrotome blade. Then stricture was cut with a sharp blade at 12 ' O clock position by using an upward movement of the

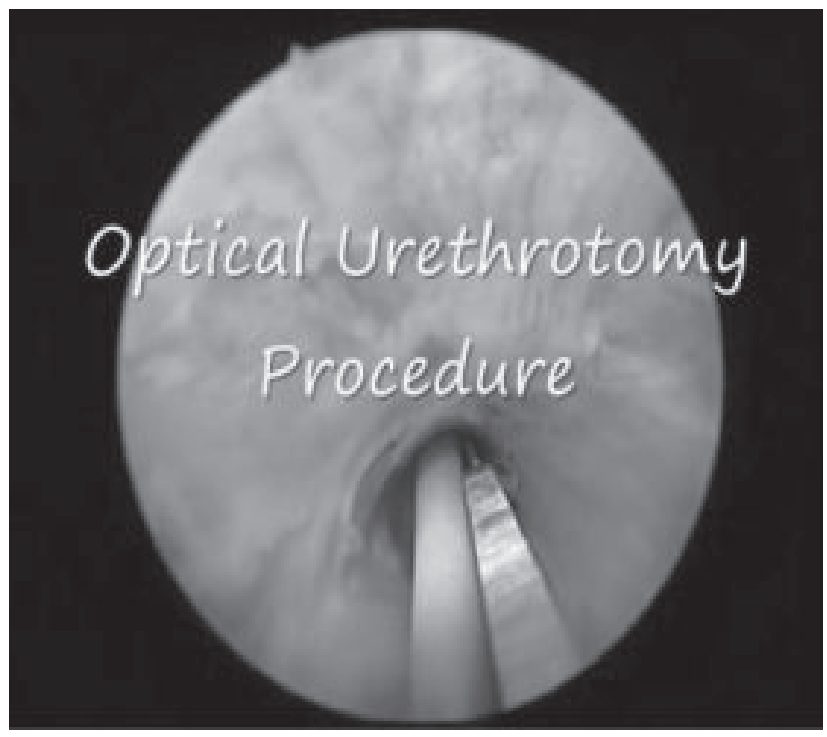

Fig.1 : Optical urethrotomy in progress bladeand cutting continued until all of the fibers of the stricture was divided along its entire length and depth.

After internal urethrotomy cystoscopy was performed to inspect the bladder for any abnormalities. In the same way laser urethrotomy was done using 365urn contact end firing quartz laser fiber passing through urethroscope produced by 80 W SPHINX HolmiumYAG Laser, a medical pulse laser and if needed ablation of scar tissue was also done in some cases. The average laser energy of $15 \mathrm{~W}$ withpulse energy $0.5-1.2 \mathrm{~J}$. repetition (frequency) $15-20 \mathrm{~Hz}$ and duration 700us was used. After complete incision of the stricture area while sparing healthy mucosa and confirming free passage of the cystoscope into the bladder a 16 FR Foley urethral catheter was left in place. Catheter was usually removed 24-72 hrs after the procedure. All patients were advised to attend at urology OPD at 3 months, 6 months and 1 year after operation. Follow-up protocol includes-history(about the flow and frequency of urine, difficulty in micturition and erectile dysfunction) ,physical examination urinalysis, culture and sensitivity, Uroflowmetry ,RGU \& MCU( at 6 months and 1 year after the operation). Clean intermittent self catheterization (CISC) with 14 FR nelaton catheter was advised if flow rate was $<15 \mathrm{ml} / \mathrm{s}$ during follow up visit. Outcome of both groups were compared on the basis of - recurrent stricture formation, peroperative $\&$ postoperative complications, duration of catheterization \& hospital stay, operative time, results of urinalysis, uroflowmetry, retrograde urethrogram and micturiting cystourethrogram, Recurrent stricture

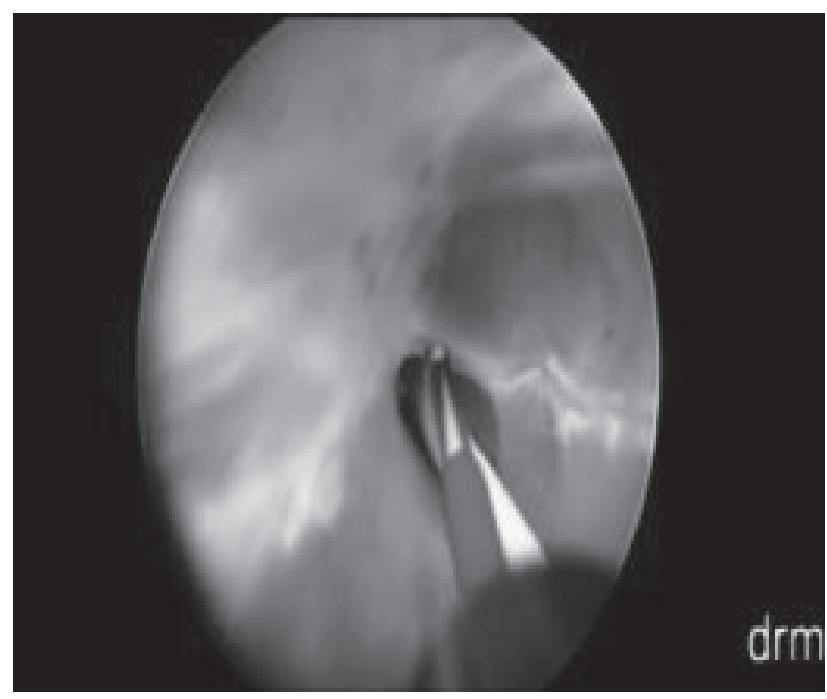

Fig.2: Laser urethrotomy in progress with guide wire in situ 
formation was considered as failure and no stricture as success seen by retrograde urethrogram .Good urine flow rate $(\mathrm{Qmax}>15 \mathrm{ml} / \mathrm{s})$ was considered as success and poor urine flow rate $(\mathrm{Qmax}<15 \mathrm{ml} / \mathrm{s})$ as failure.Numbers of peroperative \& postoperative complications were interest. Data were processed and analysed with the help of computer software SPSS version 20. The appropiate test statistics(eg. Chi-square $\left(\chi^{2}\right)$ or Fisher's Exact Probability Test and Student's tTest) were used to analyse the data.

\section{Results}

At one year of evaluation leaving 28 patients in GroupA and 30 in Group-B(after lost in follow-up) for final outcome analysis. The outcome of considered and maximum number of complications are taken as failure and minimum number of complications as success. Duration of postoperative catheterization and postoperative hospital stay were considered as important factors. The clinical history of the patients, physical examination findings and relevant investigations and operative and postoperative follow up results were recorded on a structured questionnaire which addressed all the variables of two groups were evaluated and compared. The mean ages of Group-A and Group-B were $35.9 \pm 11.2$ and $39.5 \pm 12.3$ years respectively. The lowest highest ages ranged from 1570 years $(p=0.227)$. Comparison of baseline investigations findings shows the mean baseline Qmax at uroflowmetry of Group-A was observed to be 5.93 \pm 2.90 and that of Group-B was $6.01 \pm 2.94 \mathrm{ml} / \mathrm{sec}(\mathrm{p}=$ 0.912). Peroperative complications like bleeding, extravasations of irrigating fluid, false passage and broken knife all were encountered by Group-A compared none by Group-B. However, only bleeding demonstrates its significant presence in Group-A (21.9\%). Total peroperative complications in the former group were $31.3 \%$ compared none in the latter group. Postoperative complications between groups incidences of UTI were higher in Group-A than those in Group-B, although the differences were not statistically significant $(p=0.500)$. Complications like bleeding, haematoma, penile oedema and erectile dysfunction were found only in Group-A. Epididymitis and incontinence were equally distributed between groups. Total postoperative complications were significantly higher in Group-A ( $p=0.002)$. The mean operation time was found to be significantly less in
Group-A compared to that in Group-B (16.7 minutes vs. 21.1 minutes, $p=0.0008)$. The mean duration of postoperative catheterization was observed to be much higher

in Group-A than that in Group-B ( $\mathrm{p}=0.000008)$. The average hospital stay was significantly higher in Group-A (3.56 \pm 1.24 days) than that in Group-B (2.31 \pm 0.47 days) ( $p=0.0000$ No difference was observed between the groups in terms of Qmax at discharge and 3,6 , and 12 months after operation.

Table I : Comparison of peroperative complications between groups

\begin{tabular}{lcc}
\hline Peroperative & \multicolumn{2}{c}{ Group } \\
\cline { 2 - 3 } variables* & $\begin{array}{c}\text { Group- A } \\
(\mathrm{n}=32)\end{array}$ & $\begin{array}{c}\text { Group-B } \\
(\mathrm{n}=32)\end{array}$ \\
\hline Bleeding & $7(21.9)$ & 00 \\
Extravasations of & $2(6.3)$ & 00 \\
irrigating fluid & & \\
False passage & $1(3.1)$ & 00 \\
Broken knife & $1(3.1)$ & 00 \\
Total complications & $11(31.3 \%)$ & 00 \\
\hline
\end{tabular}

*Figures in the parentheses denote corresponding \%.

Table-II: Comparison of postoperative complications between groups

\begin{tabular}{lccc}
\hline Postoperative & \multicolumn{2}{c}{ Group } & $\begin{array}{c}\mathrm{p}- \\
\text { complications* }\end{array}$ \\
\cline { 2 - 3 } & $\begin{array}{c}\text { Group-A } \\
(\mathrm{n}=32)\end{array}$ & $\begin{array}{c}\text { Group-B } \\
(\mathrm{n}=32)\end{array}$ & value \\
\hline Bleeding & $2(6.3)$ & 00 & - \\
Haematoma & $3(9.4)$ & 00 & - \\
Penile oedema & $2(6.3)$ & 00 & - \\
UTI & $6(18.8)$ & $5(15.6)$ & 0.500 \\
Epididymo orchitis & $1(3.1)$ & $1(3.1)$ & 0.754 \\
Incontinence & $1(3.1)$ & $1(3-1)$ & 0.754 \\
Total postoperative & $15(46.8)$ & $7(21.8)$ & 0.002 \\
complications & & & \\
\hline
\end{tabular}

*Figures in the parentheses denote corresponding $\%$. 


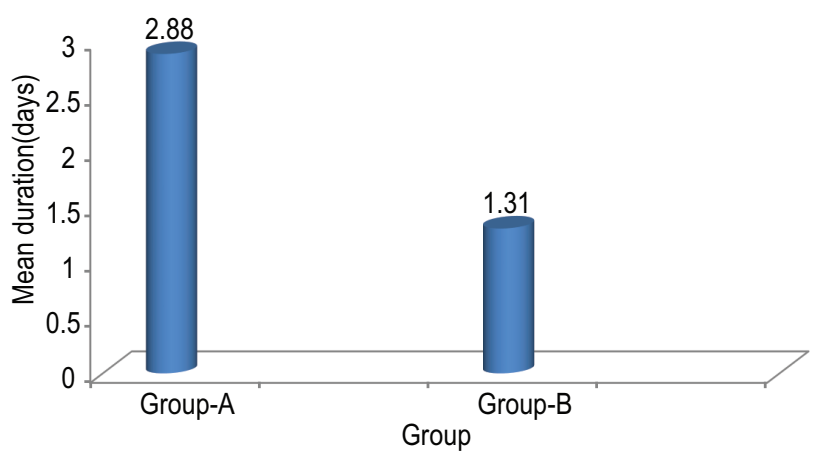

Fig.-3: Duration of postoperative catheterization

The mean Qmax at discharge was nearly $20 \mathrm{ml} / \mathrm{sec}$ which decreased to around $17 \mathrm{ml} / \mathrm{sec}$ after 1 year of evaluation. The Group-B demonstrated a higher success rate compared to Group-A (90.3\% vs. $83.9 \%$ respectively) at 6 months of evaluation. However, the difference did not turn to significant $(p=0.354)$.

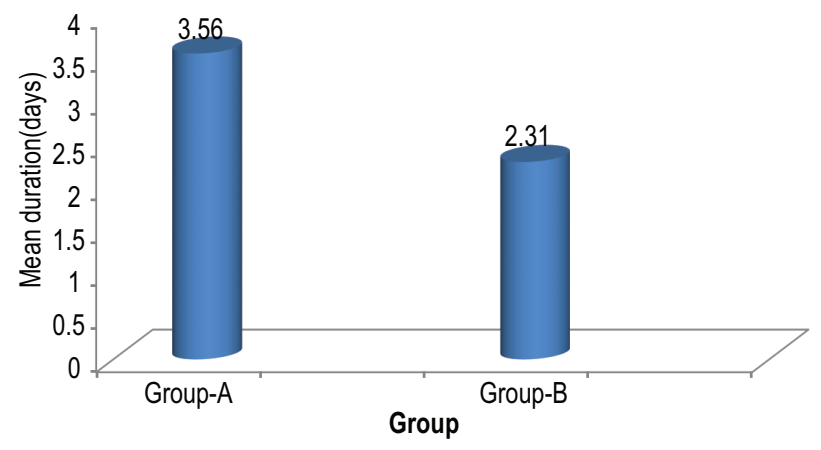

Fig.-4: Duration of postoperative hospital stay

Table-III :Comparison of final outcome at 1 year of follow up

Group pGroup-A Group-B value $(\mathrm{n}=28) \quad(\mathrm{n}=30)$

Final outcome

\begin{tabular}{lccc} 
Success & $22(78.6)$ & $26(86.7)$ & 0.320 \\
Failed & $6(21.4)$ & $4(13.3)$ & \\
\hline
\end{tabular}

Figures in the parentheses denote corresponding \%, \# Data were analysed using Chi-square $\left(\chi^{2}\right)$

Comparison of final outcome between groups at 1 year of evaluation following operation-s the success rates in Group-A and Group-B further decreased to $78.6 \%$ and $86.7 \%$ respectively, although the difference between the two groups did not reach the level of significance $(p=0.320)$.

\section{Discussion}

This study was carried out prospectively to find out the outcome of direct vision internal urethrotomy using cold knife (optical) and HO:YAG laser (laser) for the treatment of recurrent stricture urethra following perineal anastomotic urethroplasty for PUDD in the department of urology, Dhaka Medical College Hospital, Dhaka. Initially total 64 patients were selected for the study, of them 32 were randomly assigned for Group-A, treated by optical urethrotomy and 32 to Group-B, treated by Ho:YAG laser urethrotomy. Six (6) patients were lost from follow up; among them 2 patients were lost at 6 months, 1 from each group \& 4 patients at 1 year, 3 from Group-A \& 1 from Group-B. After 1 year of follow up 28 patients in Group-A and 30 patients in Group-B were available for evaluation of final outcome. All variables of interest are discussed chronologically.The demographic and baseline characteristics included in this study were almost identical between two groups. Age ranges were equal (15-70ys) and age distribution was almost homogenously distributed in both the groups $(\mathrm{p}>0.05)$. The baseline preoperative uroflowmetry demonstrated that mean baseline Qmax in Group-A was 5.93 $\pm 2,90$ $\mathrm{ml} / \mathrm{s}$ and that of Group-B was $6.01 \pm 2.94 \mathrm{ml} / \mathrm{s}$. It was almost identical statistically. 32 patients treated by $\mathrm{Kamp}^{7}$ with laser urethrotomy with preoperative Qmax $12.45 \mathrm{ml} / \mathrm{s}$ which was much higher than this study.Complications encountered following optical urethrotomy and laser urethrotomy were compared. Overall peroperative complications are $31.3 \%$ (11 cases) among the patients treated with optical urethrotomy and none with laser urethrotomy. Per-operative bleeding was the most common complication. Seven patients (21.9\%) developed bleeding during optical urethrotomy, 5 patients had only minor bleeding which was stopped after catherization and compressive penile dressing. Only 2 patients continued to bleed postoperatively and one patient needed blood transfusion.Eextravasation of irrigating fluid occurred in one patient and one patient developed false passage during optical urethrotomy but normal passage could be established after repositioning the guide wire. These two patients postoperatively developed penile oedema which was diminished quickly. Six patients in GroupA and 5 patients in Group-B developed postoperative urinary tract infection despite the use of prophylactic antibiotics and they were treated successfully by using 
culture specific antibiotics. One patient in each group developed epididymitis which was treated by antibiotics, analgesic and scrotal support. One patient in each group developed urinary incontinence after removal of catheter. But it was

only transient and both patients gained control within short time. Kamp ${ }^{7}$ noted 5\% patients developed postoperative urinary tract infection after laser urethrotomy.. Smith ${ }^{6}$ treated 39 patients by optical urethrotomy. One patient had severe postoperative bleeding and required blood transfusion, one patient developed a secondary haemorrhage and readmitted for transfusion. Two patients developed postoperative pyrexia with urinary infection and one patient developed extravasation of irrigating fluid. Giannokopoulos ${ }^{8}$ treated 80 patients with optical urethrotomy (40 patients) and optical urethrotomy + transurethral resection (40 patients) and reported cpididymitis (7 patients), scrotal oedema (11 patients), pcrineal haematoma (8 patients), and extravasation of irrigating fluid (16 patients) which were much higher than this series. On the other hand several investigators showed no intra-operative complication during laser urethrotomy. The operative time needed for optical urethrotomy was less than that for laser urethrotomy (16.7 minutes vs. 21.1 minutes). Although it found statistically significant, the actual time difference was only 4-5 minutes. Mean operative time was 21 minutes (range 5-45 minutes) for laser urethrotomy which was similar to this study. After optical urethrotomy catheter was usually removed between 1 to 3 days postoperatively. In 2 patients it was removed on $5^{\text {th }}$ postoperative day and in 2 patients on $7^{\text {th }}$ day due to development of complications (haematoma, penile oedema). On the other hand in laser group all catheters were removed within 48 hours. Smith et al kept catheter indwelling for 24 to 72 hours only.After laser urethrotomy Kamp et al left catheter for 4 days. Uroflowmetry was done for every patient during discharge and all patients showed good flow at discharged (Qmax $>15 \mathrm{ml} / \mathrm{sec}$ ) and average Qmax was nearly $20 \mathrm{ml} / \mathrm{sec}$ in both groups. Postoperative urethrogram (RGU\&MCU) was done at 6 months and 1 year of follow-up. Postoperative uroflowmetry and urethrography (RGU\&MCU) showed no significant difference between two groups. Recurrent stricture was considered when Qmax was $<15 \mathrm{ml} / \mathrm{sec}$ and urethrography (RGU \& MCU) showed narrowing of urethral lumen. Recurrence of stricture was evaluated at 6 months and at 1 year. At 6 months 31 patients in both groups were available for evaluation. In GroupA there were 5 cases $(16 \%)$ of recurrent strictures and $84 \%$ success rate. And in Group-B, 3 cases $(10 \%)$ of recurrent strictures with success rate of $90 \%$.

During final evaluation at 1 year, 28 patients in GroupA and 30 patients in Group-B were available. In GroupA there was $78.6 \%$ success rate with $21.4 \%$ (6 cases) recurrent strictures. On the other hand in group-B there was $86.7 \%$ success rate with $13.3 \%$ (4 cases) recurrent strictures. Although there was a higher rate of recurrent strictures in Group-A than that in Group-B, but it was not statistically significant.

Hans Sachse in June 1971 conducted his first optical urethrotomy with the prototype of a cold knife urethrotome. He treated 34 strictures with optical internal urethrotomy, catheter kept for 10 to 14 days and reported $80 \%$ success rate. Following Sachse optical urethrotomy, it has been widely used as the treatment of choice for urethral stricture throughout the world with short term success rate of 68 to $90 \%{ }^{6}$. These were very similar to this study.On the other hand laser urethrotomy was done and reported by several authors with a success rate of 69 to $90 \% 9,7,10$. These were also very similar to this study.

So, from above discussions it is clear that laser urethrotomy is a better option for the treatment of recurrent stricture urethra following perineal anastomotic urethroplasty for PUDD .

\section{Conclusion}

From the present study it can be concluded that laser urethrotomy is better

than Optical urethrotomy for the treatment of recurrent stricture urethra following perineal anastomotic urethroplasty done for posterior urethral distraction defect when per-operative \& postoperative complications, duration of catheterization and postoperative hospital stay are considered. .

\section{Limitations of the study}

It was done on a small group of patients,in a single centere and study period was short

\section{Conflict of interest: Nothing declared}

Ethical issue : Ethical committee of Dhaka Medical College \& Hospital recommended to Faculty of postgraduate Medical Science and Research ,University of Dhaka, for acceptance of the title for research study. 


\section{References}

1. Cooperberg MR., McAninch JW., Alsikafi NF., Elliott SP. (2007)'Urethral reconstruction for traumatic posterior urethral disruption: outcomes of a 25-year experience'. J Urol. ,178,pp.2006-10

2. Koraitim MM.(2003) 'Failed posterior urethroplasty: Lessons

3. Jordan GH., Schlossberg SM., Devine CJ.(2000) 'Surgery of the penis and urethra' In. Campbell's Urology, Vol-4, $8^{\text {th }}$ edition, edited by Walsh PC., Retik AB., Vaughan DE. Jr.,Wein AJ., W.B. Saunders, Philadelphia, pp. 3887-3953

4. Albers P., Fichter J., Bruhl P., Muller SC.(1996). 'Long-term results of internal urethrotomy', J Urol, 156(5),pp. 1611-4.

5. Vicente J., Salvador J., Caffaratti J. (1990)' Endoscopic Urethrotomy versus Urethrotomy plus Nd-YAG Laser in the Treatment of Urethral Stricture'. Eur Urol, 18pp.166-168.

6. Smith JA., and Dixon JA.(1984) 'Neodymium: YAG laser treatment of benign urethral strictures', J Urol, 131pp.1080-1081.
7. Kamp S., Knoll T., Osman MM., Kohnnnn KU., Michel MS. and Alken P.(2006)`Low-power Holmium: YAG Laser Urethrotomy for Treatment of Urethral Stricrures: Functional Outcome and Quality of Life'. J Endourol,20(1)pp. 38-41.

8. Giannakopbulos X., Gremmeniatis E., Gartzios A., Tsoumanis P., Kammenos A.(1997),'Sachse Urethrotomy versus Endoscopic Urethrotomy plus Transurethral resection of the fibrous callus (Guillemin's technique) in the treatment of urethral stricture, Urology, 49pp.243-247.

9. Hanash KA, Aslam M, Mokhtar AA, Merdad T, Al-Zahrani H, Al-Ghamdi A.( 2003), 'Comparative long term results of first line surgical therapies for bulbar and posterior urethral strictures'. AUJ,1pp. 41-46.

10. Zbigniew Jab ${ }^{3}$ onowski, M.D., Ph.D., Robert $\mathrm{Ke}^{2}$ dzierski, M.D., Ph.D.,Eugeniusz Mie'ºs' , and Marek Sosnowski(2010) 'Comparison of Nd-YAG laser treatment with cold knife endoscopic Incision of urethral strictures in male patients', JPhotomedicine and Lase Surgery, 28(2)pp.2 